\title{
ANÁLISE DE DECISÃO MULTICRITÉRIO PARA IDENTIFICAÇÃO E PRIORIZAÇÃO DE ESTRATÉGIAS DE SEGURANÇA PÚBLICA DURANTE A PANDEMIA DA COVID-19 ${ }^{1}$
}

\author{
Maria Camyla Gonçalves Wanderley ${ }^{\mathrm{a}}$, João Paulo Santos Aragão ${ }^{\mathbf{a}^{*}}$ \\ ${ }^{a}$ Centro Acadêmico do Agreste, Núcleo de Tecnologia \\ Universidade Federal de Pernambuco - UFPE, Caruaru-PE, Brasil
}

Recebido 03/08/2020, aceito 02/10/2020

\begin{abstract}
RESUMO
A segurança pública é um direito e dever de todos, corresponde à proteção do cidadão e do patrimônio, prevenindo e controlando manifestações da criminalidade e da violência. Contudo, a criminalidade tende a agravar-se em períodos de instabilidade, como a pandemia causada pela COVID-19. Nesse sentido, é importante que a esfera pública identifique e priorize ações estratégicas que assegurem a segurança dos cidadãos. Dessa forma, este estudo teve como objetivo identificar e priorizar estratégias de segurança pública a fim de reduzir a criminalidade durante o período de isolamento social ocasionado pela COVID-19. Para isso, fez-se uso das metodologias Value Focused Thinking e FITradeoff de ordenação. Por meio de um estudo de caso em um município do interior do estado de Pernambuco, foi possível recomendar ao decisor as ações que devem ser encaradas como prioridade para garantia da segurança pública. A ação voltada para a segurança da mulher foi considerada como prioritária.
\end{abstract}

Palavras-chave: Segurança pública, Decisão multicritério, Value focused thinking, FITradeoff de ordenação.

\begin{abstract}
Public security is a right and duty of all, it corresponds to the protection of citizens and property, preventing and controlling manifestations of crime and violence. However, crime tends to worsen in periods of instability, such as the pandemic caused by COVID-19. In this sense, it is important that the public sphere identifies and prioritizes strategic actions that ensure the safety of citizens. Thus, this study aimed to identify and prioritize public security strategies to reduce crime during the period of social isolation caused by COVID-19. For this work, the methodologies Value Focused Thinking and FITradeoff of ranking were used. Through a case study in a city from the interior of Pernambuco, it was possible to recommend to the decision maker the actions that should be a priority to guarantee public security. Action aimed at the safety of women was considered as a priority.
\end{abstract}

Keywords: Public security, Multicriteria decision, Value focused thinking, FITradeoff for ranking.

*Autor para correspondência. E-mail: aragaopaulojoao@gmail.com

DOI: 10.4322/PODes.2021.003 


\section{Introdução}

Conforme o Art. 144 da Constituição Federal, "a segurança pública, dever do Estado, direito e responsabilidade de todos, é exercida para a preservação da ordem pública e da incolumidade das pessoas e do patrimônio [...]" (BRASIL, 1988). A segurança pública, ainda de acordo com o art. 144 é preservada por meio de órgãos estatais, sendo estes: polícia federal, rodoviária, ferroviária, civil, militar e corpo de bombeiros militares. Portanto, segurança pública corresponde à proteção do cidadão e do patrimônio, prevenindo e controlando manifestações da criminalidade e da violência, garantindo o exercício pela cidadania.

Contudo, apesar de ser garantia e do direto à segurança pública, a percepção de insegurança e o aumento da violência no cenário brasileiro é crescente. Segundo os dados do Sistema Nacional de Informações de Segurança Pública (Sinesp 2020), disponibilizados pelo Ministério da Justiça e Segurança Pública, no Brasil, no ano de 2019, o número de homicídios foi de aproximadamente 44.102, desse total, os tipos de crimes incluem homicídio doloso, lesão corporal seguida de morte e latrocínio.

Portanto, nesse cenário caracterizado por um alto índice de criminalidade, a adoção de políticas públicas que aumentem a segurança deve ser encarada como prioridade. As políticas públicas podem ser vistas como uma maneira de prevenir, reduzir e mitigar a criminalidade e a violência por meio de ações e programas que defendam diretamente ou indiretamente a vida dos cidadãos, independentemente de sua idade, classe social, gênero ou orientação sexual.

Segundo especialistas, a criminalidade e a violência tendem a agravar-se em períodos de instabilidade, como crises financeiras. De acordo com o Anuário Brasileiro de Segurança Pública (2019), em momentos de crises, o debate nacional fica restrito à agenda econômica e, com isso, afasta-se o olhar da esfera pública de outros temas e prioridades, como o tema de segurança pública. Nesse contexto, destaca-se o cenário atual de instabilidade global devido ao novo coronavírus.

Em 11 de março de 2020, a Organização Mundial de Saúde (OMS) declarou que o novo coronavírus (COVID-19) havia sido caracterizada como uma pandemia (OMS, 2020). Na América Latina o primeiro caso suspeito e confirmado da COVID-19 foi no Brasil (RodriguezMorales et al., 2020). Os primeiros passos para conter o surto da COVID-19 no país tiveram início ainda no primeiro semestre, contudo a doença vem se espalhando muito rapidamente no Brasil (Itiki e Chowdhury, 2020). Nesse cenário o governo brasileiro tem buscado soluções de saúde pública para minimizar os danos causados pela pandemia. Entretanto, além destas soluções outros aspectos devem ser analisados e mitigados, como por exemplo o desemprego, a educação, a criminalidade e a violência doméstica.

No âmbito de violência doméstica, de acordo com o Anuário Brasileiro de Segurança Pública (2020b), observou-se um aumento de 34\% nos registros de denúncias na Central de Atendimento à Mulher durante os meses de março e abril de 2020 em comparação com o mesmo período em 2019. Houve também um crescimento de cerca de $22 \%$ no número de feminicídios e $6 \%$ no quantitativo de homicídios de mulheres, tudo isto em apenas dois meses de isolamento social devido a pandemia da COVID-19.

Assim, é importante que a esfera pública priorize estratégias que assegurem a segurança pública ao mesmo tempo que garanta a continuidade dos serviços públicos durante o período da pandemia da COVID-19, a qual traz consigo inúmeras implicações e desafios na vida da sociedade. Contudo, priorizar essas ações em meio a um ambiente muitas vezes caracterizado por falta de informações e tempo reduzido, pode não ser uma tarefa trivial. Além disso, definir qual estratégia é prioritária pode envolver múltiplos critérios que, em geral, tendem a ser conflitantes entre si.

Desse modo, este estudo tem como objetivo definir e priorizar ações estratégicas de segurança pública a fim de reduzir a criminalidade em um município do interior do estado de Pernambuco, Brasil, considerando o momento atual de pandemia ocasionada pela COVID-19. Para se conseguir ao objetivo proposto, fez-se uso de um método de estruturação de problemas, o Value-Focused Thinking (VFT), para auxílio na definição das ações de segurança, bem como a utilização do método multicritério de apoio à decisão, o método FITradeoff para ordenação 
das ações. Com isso, tornou-se possível recomendar ao decisor do problema ações estratégicas que devem ser encaradas como prioridade para garantia da segurança pública do município.

Além dessa Seção, este estudo está dividido da seguinte maneira, a saber: na Seção 2 o os métodos de suporte à decisão VFT e FITradeoff para ordenação são apresentados; na Seção 3 a metodologia utilizada é descrita; já na Seção 4 evidencia-se o estudo de caso e aplicação da metodologia; na sequência, na Seção 5 as discussões são apresentadas e, por fim, na Seção 6, tem-se as considerações finais.

\section{Métodos de Suporte à Decisão}

\subsection{Value Focused Thinking}

Em meados da década de 1970, em resposta, principalmente, às dificuldades e limitações do uso exclusivo de ferramentas quantitativas (PO hard), surgiram os métodos de estruturação de problemas (Problem Structuring Methods - PSM), classificados como ferramentas da PO soft (Smith e Shaw, 2019). Na visão de Belton e Stewart (2002), independentemente do nível de complexidade, é necessário um PSM para melhor compreensão do problema. Entre os métodos de estruturação mais conhecidos destaca-se o Value Focused Thinking (VFT).

Proposto por Keeney (1992), o VFT é uma metodologia que observa e destaca os objetivos mais sólidos do decisor de modo que estes não fiquem em segundo plano. Seu processo de construção advém inicialmente da explicitação de valores, onde deve haver a identificação dos objetivos. Os objetivos podem ser classificados em três categorias: objetivos fundamentais, que são as motivações reais e essenciais para ocorrer a decisão; objetivos meios, são aqueles que auxiliam os objetivos fundamentais a serem alcançados; objetivos estratégicos, referem-se aos objetivos maiores do decisor, ou seja, seus objetivos finais (Keeney, 1994).

O VFT revela objetivos que até então não tinham sido considerados, permitindo ao decisor criar alternativas de decisão e melhorar a comunicação entre os stakeholders, tendo em vista que os objetivos são exibidos em uma rede de objetivos meio e fim de forma lógica (Keeney, 1994; Alencar et al., 2017).

O VFT é uma metodologia que frequentemente é utilizada com um método multicritério de apoio a decisão. Na literatura é possível verificar estudos cuja estruturação de problemas deu-se a partir do VFT, como os estudos de Höfer e Madlener (2020), Poleto et al. (2020), Almaian et al. (2016), Merrick e Grabowski (2014), Morais et al. (2013), e Lesinski (2015).

\subsection{FITradeoff para Ordenação}

Desenvolvido por de Almeida et al. (2016), o método FITradeoff (Flexible and interactive tradeoff) apresenta destaque no universo dos métodos MCDA, pois ele é interativo, flexível e é baseado no método clássico de tradeoff, o qual possui uma forte base axiomática para elicitação de pesos (Keeney e Raiffa, 1976). Além disso, utiliza o conceito de informações parciais, o que minimiza o esforço cognitivo do decisor, pois o número de perguntas e o nível de dificuldade delas são reduzidas. Isto ocorre pois, conforme evidenciado por de Almeida et al. (2016), o FITradeoff busca estabelecer relações de preferencias estrita entre as consequências e não as indiferenças, as quais são consideradas no tradeoff clássico.

É importante destacar que no processo de solução de problemas utilizando o FITradeoff, o decisor responde perguntas que consideram tradeoffs entre as consequências, em que deve declarar relações de preferência estrita entre elas. Com isso, as informações das respostas do decisor são utilizadas para atualizar o espaço de pesos a cada iteração (de Almeida et al., 2016).

Desenvolvido originalmente para solucionar problemas de escolha, este método tem sido amplamente aplicado na literatura para resolver problemas em diferentes contextos, como os estudos de Gusmão e Medeiros (2016), Frej et al. (2017), Carrillo et al. (2018), Fossile et al. (2020) e Pergher et al. (2020).

Mais tarde, Frej et al. (2019) apresentaram uma nova versão do método, dessa vez desenvolvida para atender a problemática de ordenação. A principal diferença entre 0 
FITradeoff de ordenação e o de escolha, é que o FITradeoff de ordenação usará um modelo de programação linear (PPL) para cada par de alternativas a fim de tentar encontrar relações de dominância, ao invés de encontrar a alternativa potencialmente ótima como no método de escolha.

Portanto, a cada ciclo, o seguinte PPL é efetuado para cada par de alternativas $\left(A_{i}, A_{k}\right)$ (Frej et al. 2019), conforme as equações (1) a (5):

$$
\max D\left(A_{i}, A_{k}\right)=\sum_{j=1}^{m} w_{j} v_{j}\left(A_{i}\right)-\sum_{j=1}^{m} w_{j} v_{j}\left(A_{k}\right)
$$

Sujeito a:

$$
\begin{gathered}
w_{1}>w_{2}>\ldots>w_{m} \vee \sum_{j=1}^{m} w_{j}=1 \\
w_{j} v_{j}\left(x_{j}^{\prime}\right)>w_{j+1} j=1 \text { para } m-1 \\
w_{j} v_{j}\left(x_{j}^{\prime \prime}\right)>w_{j+1} j=1 \text { para } m-1 \\
w_{j} \geq 0, j=1 \ldots m
\end{gathered}
$$

Onde, m é o número total de critérios; $w_{j}$ são as constantes de escala para cada critério $j$; $A_{i}$ e $A_{k}$ são as alternativas a serem comparadas; a função valor é representada por $v_{j}$; e $x_{j}$ são os valores intermediários a serem avaliados.

À medida que o decisor responde a perguntas no FITradeoff, mais restrições são obtidas, de modo que o espaço de peso fica mais estreito. Com uma matriz de dominância aos pares, é possível construir um ranking (parcial ou completo, dependendo da quantidade de informação obtida) das alternativas a cada ciclo (Frej et al., 2019).

\section{Metodologia Proposta}

A metodologia parte do pressuposto de que os atores do processo decisório, como decisor, facilitador e analista foram previamente definidos.

Dividida em 04 etapas básicas, a descrição da metodologia é representada pela Figura 1.

Figura 1: Etapas da metodologia.

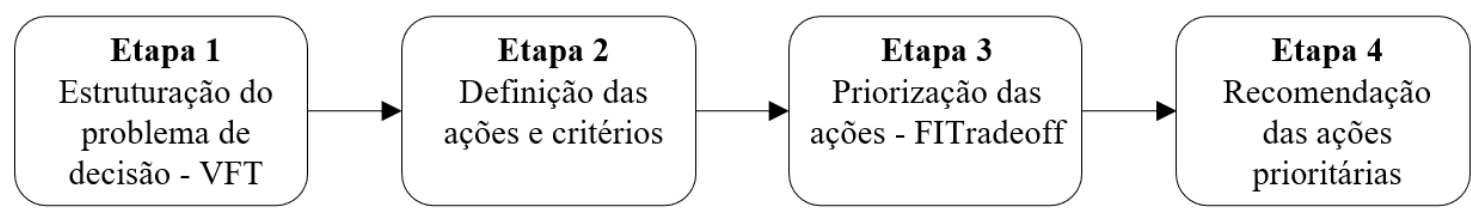

Fonte: Elaborada pelo autor.

Na Etapa 1 tem-se a estruturação do problema de decisão por meio do método VFT. A construção do VFT é dada pela definição dos objetivos (estratégicos, fundamentais e meios) para construção da hierarquia dos objetivos.

Compreendido melhor o problema e capturado as particularidades do contexto, por meio da rede de objetivos do decisor, são estabelecidas as ações estratégicas de segurança pública e os critérios de avaliação, conforme Etapa 2. As ações de segurança pública referem-se aquelas das quais o decisor deseja priorizar. Já os critérios de avaliação são aqueles que irão subsidiar a avaliação das ações. Portanto, é essencial que a definição dos critérios seja bastante discutida e 
que os critérios de avaliação estejam alinhados aos objetivos estratégicos do decisor. Além disso, os critérios devem ser claros e significativos. Também nesta etapa, as escalas de avaliação e os critérios dos objetivos devem ser evidenciadas.

Após a definição das ações de segurança pública e os critérios de avaliação, caso o decisor julgue o conjunto obtido insatisfatório ou incompleto, ele pode retornar à etapa anterior para uma maior reflexão dos valores gerados durante a construção do VFT. Mas caso o decisor sinta-se representado pelo conjunto de ações e critérios, segue-se para a Etapa 3.

Para a ordenamento das ações por meio do FITradeoff, é necessária a construção da matriz de avaliação, que consiste na avaliação das ações de segurança pública em cada critério conforme as escalas de avaliação estabelecidas. Com base nisso, o método FITradeoff de ordenação é aplicado com o auxílio do software desenvolvido pelo Centro de Desenvolvimento em Sistemas de Informação e Decisão (CDSID), disponível em http://fitradeoff.org/download/.

A escolha do método FITradeoff de ordenação deu-se, inicialmente, a partir da necessidade de priorizar as ações de segurança pública, isso torna-se possível por meio da geração de um ranking; racionalidade compensatória do decisor, uma vez que o decisor do problema acredita que para essa situação há tradeoffs entre os critérios; a quantidade reduzida de informações exigida por parte do decisor.

Por fim, na Etapa 4 tem-se o ordenamento das ações de segurança pública, sendo possível recomendar ao decisor as ações prioritárias. Destaca-se que caso o decisor não estivesse satisfeito com a solução apresentada, ele poderia retornar às fases anteriores e revisar alguns parâmetros.

\section{Estudo de Caso}

De acordo com a Secretaria de Defesa Social do estado de Pernambuco (SDS-PE), em 2019, o número de Crimes Violentos Letais Intencionais (CVLI) e de Crimes Violentos contra o Patrimônio (CVP) foram de 3.469 e 79.322, respectivamente. De janeiro a junho de 2020, o estado registrou 1.961 casos de CVLI e 28.087 casos de CVP (SDS-PE, 2020).

Para essas estatísticas, o estado de Pernambuco é dividido em três regiões: capital, região metropolitana e interior. O município objeto de estudo localiza-se na região do interior. Essa, por sua vez é responsável por 1131 dos casos de CVLI e 9790 dos casos de CVP do estado no período de janeiro a junho de 2020.

Motivado pelos elevados números CVLI e CVP da região, associados a uma redução orçamentária e isolamento social causados pela pandemia da COVID-19, o município buscou adotar medidas que assegurassem a segurança pública. Contudo, as ações já adotadas pelo município não eram suficientes para o atual cenário de pandemia. Dessa forma, a gestão pública municipal, representada pelo gestor de segurança pública, esse designado pelo município como decisor do problema, buscou priorizar ações consideradas estratégicas que auxiliassem na adoção e manutenção de medidas efetivas de segurança pública no município no cenário da COVID-19. Portanto, a metodologia representada pela Figura 1 foi aplicada.

\subsection{Estruturação do Problema de Decisão}

O processo de aplicação do VFT deu-se a partir de uma entrevista não-estruturada com o decisor com o intuito de verificar a sua percepção sobre o problema. A identificação dos objetivos requer muita criatividade e reflexão. De acordo com Keeney (1996), questionamentos que estimulem a identificação dos objetivos de maneira a incitar o decisor a levantar elementos que constituem o resultado final para a situação de decisão, são questionamentos importantes. Além desses, deixar o questionamento específico auxilia que o objetivo do decisor se torne cada vez mais claro.

Nesse sentido, questionamentos do tipo "Quais políticas você acha necessário para redução da criminalidade no seu município?", "Qual ação de segurança é importante para garantir a o bem-estar da população durante a pandemia?", entre outros, foram feitos ao decisor para uma melhor compressão do problema e identificação dos objetivos. Com isso, tornou-se 
possível identificar os objetivos do decisor e construir a hierarquia. A rede hierarquizada dos objetivos do decisor é representada pela Figura 2.

Figura 2: Rede de objetivos do decisor.

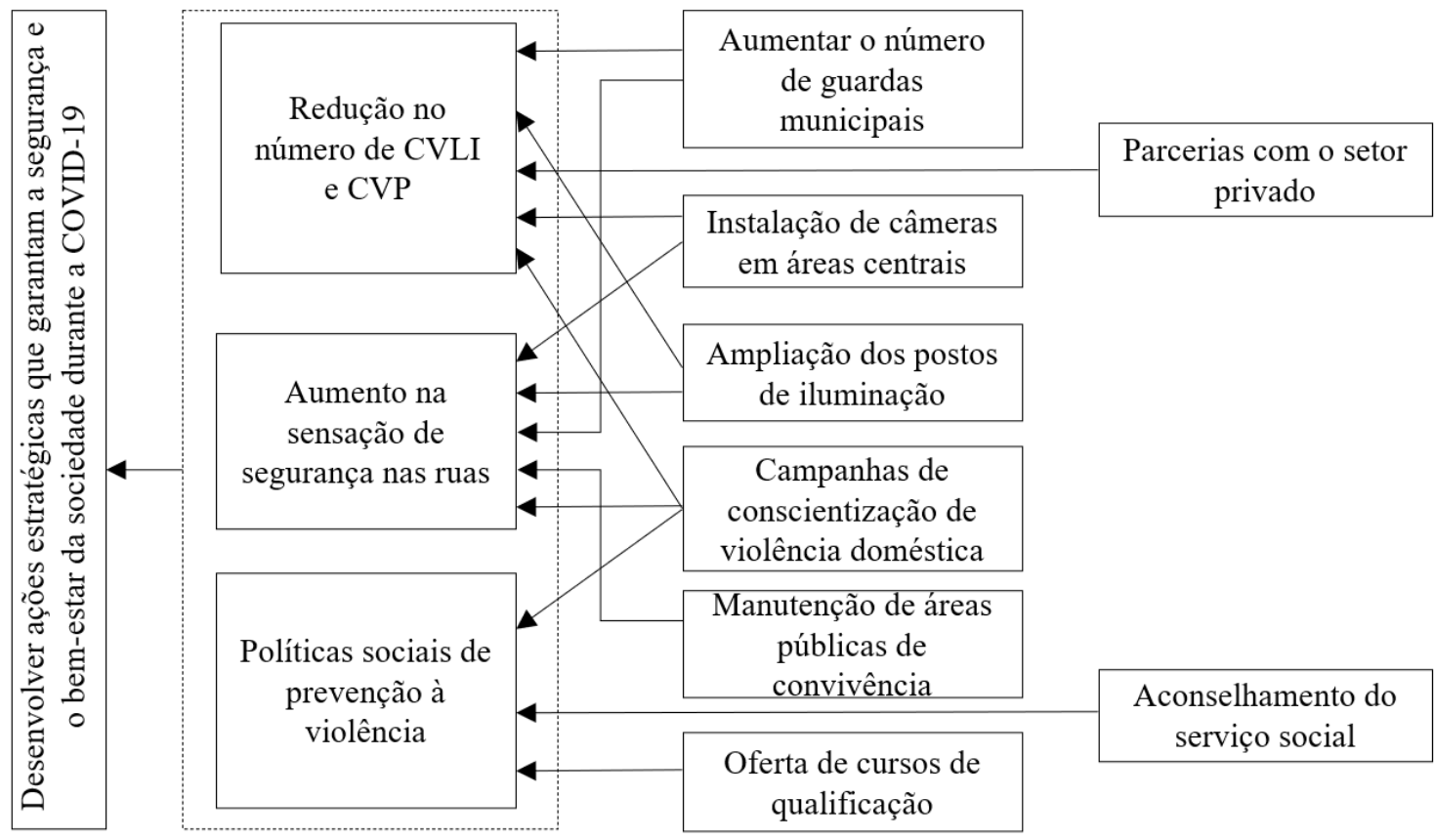

Fonte: Elaborada pelo autor.

Após a eliminação dos objetivos redundantes, conforme a Figura 2, foi obtido um objetivo estratégico, rotulado como "Desenvolver ações estratégicas que garantam a segurança e o bem-estar da sociedade durante a COVID-19", três objetivos fundamentais (redução do número de CVLI e CVP, aumento da sensação de segurança nas ruas e políticas sociais de prevenção à violência) e oito objetivos meios. Destaca-se que há objetivos meios que permitem $\mathrm{o}$ alcance de mais de um objetivo fundamental.

Aplicada a metodologia VFT, tornou-se possível a definição das ações de segurança pública e dos seus respectivos critérios de avaliação.

\subsection{Definição das Ações e Critérios}

Com base na rede de objetivos do decisor, tornou-se possível definir as ações estratégicas como políticas públicas de segurança para o município.

De acordo com Keeney (1996), as primeiras alternativas que vêm à mente em uma determinada situação são as óbvias, as que já foram utilizadas em situações semelhantes e as que estão prontamente disponíveis. Contudo, alternativas verdadeiramente diferentes permanecem ocultas em outra parte da mente humana. É necessário um pensamento profundo e persistente para trazê-los à tona.

Portanto, a partir do foco no valor, ou seja, foram criadas alternativas que melhor atingissem os valores especificados para a situação de decisão. Tanto os objetivos qualitativos quanto as declarações quantitativas de valores (por exemplo, prioridades) foram sistematicamente investigados para iniciar o pensamento criativo.

A partir da rede de objetivos do decisor, os objetivos fundamentais listam todos os aspetos das consequências que são importantes em uma situação de decisão. Assim, pensar em como melhor atingir esses objetivos faz com que surja novas alternativas.

Logo, ao fim desse processo, um conjunto de 08 ações de segurança pública foram definidas. As ações são apresentadas no Quadro 1. 
Quadro 1: Ações de segurança pública.

\begin{tabular}{|l|l|}
\hline \multicolumn{2}{|c|}{ Ações } \\
\hline a1 & Criação de unidade especializada para investigação de homicídios \\
\hline a2 & Criar grupo interinstitucional de prevenção à violência \\
\hline a3 & Criar uma central especializada de apoio à violência contra a mulher \\
\hline a4 & Aumentar o número de guardas municipais \\
\hline a5 & Manutenção de áreas públicas de convivência \\
\hline a6 & Criação de comitê de prevenção à violência nas escolas \\
\hline a7 & $\begin{array}{l}\text { Fortalecer programas de reinserção de egressos do sistema prisional na } \\
\text { cidade }\end{array}$ \\
\hline a8 & Ampliar o sistema de monitoramento de câmeras pela cidade \\
\hline
\end{tabular}

Fonte: Elaborada pelo autor.

Definidas as ações estratégicas de segurança pública, o decisor definiu um conjunto de quatro critérios de avaliação. Foram eles:

cl - Tempo de efetivação: refere-se ao tempo necessário para implantação da ação. Como escala de avaliação, o decisor optou por medir o desempenho das ações em unidade de tempo (meses).

c2 - Impacto na redução do número de homicídios: corresponde ao impacto que a adoção da ação tem na redução do número de homicídios. O desempenho do critério foi avaliado por meio de uma escala nominal de 3 pontos: (1) impacto baixo, (2) impacto moderado e (3) impacto alto.

c3 - Investimento: diz respeito ao investimento inicial necessário para implantação da ação. Por meio de estudos do órgão de compras do município, juntamente com as secretarias que possivelmente possam estar envolvidas na implantação das ações, o decisor possui uma estimativa do investimento inicial em unidades monetárias (R\$). É importante destacar que esse critério se refere apenas ao investimento inicial, portanto, custos associados a manutenção contínua das ações, como folha de pagamento de colaboradores envolvidos, por exemplo, não foram considerados.

c4 - Impacto na sensação de segurança: refere-se ao impacto gerado na sensação de segurança da população quando adotada a ação. Para esse critério o município objeto deste estudo possuía informações já coletadas por meio de uma pesquisa de opinião sobre as ações de segurança pública e seus respectivos impactos na sensação de segurança. O desempenho do critério foi avaliado por meio de uma escala nominal de 3 pontos: (1) impacto baixo, (2) impacto moderado e (3) impacto alto.

O decisor julgou o conjunto de ações e critérios suficientes, portanto, pode-se seguir com o ordenamento das ações por meio do método FITradeoff.

\subsection{Ordenação das Ações por Meio do FITradeoff}

Para aplicação do método foi necessária a construção da matriz de avaliação, visualizada na Tabela 1. A matriz de avaliação é composta por todas avaliações de cada ação $i$ de acordo com cada critério $j$. Para obtenção dessas informações, foram realizadas reuniões com o decisor, bem como foi realizada uma análise documental. O objetivo direcional de cada critério também foi definido (maximização ou minimização). 
Tabela 1: Matriz de avaliação.

\begin{tabular}{ccccc}
\hline \multirow{2}{*}{ Ações } & \multicolumn{4}{c}{ Critérios } \\
\cline { 2 - 5 } & c1 & c2 & c3 & c4 \\
\hline a1 & 4 & 1 & 12.650 & 2 \\
a2 & 3 & 2 & 3.400 & 2 \\
a3 & 2 & 3 & 5.000 & 3 \\
a4 & 4 & 3 & 21.300 & 3 \\
a5 & 6 & 2 & 35.500 & 2 \\
a6 & 2 & 1 & 4.000 & 1 \\
a7 & 1 & 3 & 4.800 & 2 \\
a8 & 4 & 2 & 3.750 & 3 \\
\hline Objetivo & Min & Max & Min & Max \\
\hline
\end{tabular}

Fonte: Elaborada pelo autor.

A aplicação do método FITradeoff foi dividida em duas fases, a primeira referiu-se à ordenação das constantes de escala (pesos) dos critérios, em que o decisor ordenou os critérios que possuem maior importância para os de menor importância. A segunda fase correspondeu a elicitação flexível do método, onde foram realizadas comparações par a par e o decisor pôde evidenciar qual consequência era preferível, se assim desejasse, ou se ele poderia ser indiferente a esta comparação.

A ordenação dos critérios do ponto de vista do decisor foi a seguinte:

$\mathrm{K}_{\text {Impacto na redução do nimero de homicidios }}>\mathrm{K}_{\text {Tempo de efetivacão da ação }}>\mathrm{K}_{\text {Impacto na sensação de segurança }}>\mathrm{K}_{\text {Investimento. }}$.

Após a ordenação, seguiu-se com sucessivos questionamentos feitos ao decisor na fase de elicitação. Entretanto, ao invés de se indicar qual critério é preferível, ele informou qual consequência hipotética, elaborada pelo software, ele teria preferência. A Tabela 2 sintetiza a aplicação do método, informando o número de ciclos, os pares de consequências analisados, qual a escolha do decisor entre elas e o número de níveis a cada ciclo.

Tabela 2: Resumo da fase de elicitação do FITradeoff.

\begin{tabular}{|c|c|c|c|c|}
\hline $\begin{array}{l}\text { Número } \\
\text { do ciclo }\end{array}$ & Consequência A & $\begin{array}{c}\text { Consequência B } \\
\text { (melhor desempenho) }\end{array}$ & Escolha & $\begin{array}{l}\text { Número } \\
\text { de níveis }\end{array}$ \\
\hline Ciclo 0 & & & Ordenação & 3 \\
\hline Ciclo 1 & $\begin{array}{l}X=2 \text { na Redução do número } \\
\text { de homicídios }\end{array}$ & Investimento & A & 4 \\
\hline Ciclo 2 & $\begin{array}{l}X=2 \text { na Redução do número } \\
\text { de homicídios }\end{array}$ & $\begin{array}{l}\text { Tempo de efetivação da } \\
\text { ação }\end{array}$ & A & 4 \\
\hline Ciclo 3 & $\begin{array}{l}X=3.5 \text { no Tempo de } \\
\text { efetivação da ação }\end{array}$ & $\begin{array}{l}\text { Impacto na sensação de } \\
\text { segurança }\end{array}$ & Indiferente & 7 \\
\hline Ciclo 4 & $\begin{array}{l}X=2 \text { no Impacto na sensação } \\
\text { de segurança }\end{array}$ & Investimento & A & 8 \\
\hline
\end{tabular}

Fonte: Elaborada pelo autor.

Assim, após 4 ciclos de elicitação, obteve-se ações a serem priorizadas. Destaca-se que durante o processo de elicitação o decisor se apresentou indiferente uma única vez, na $3^{\mathrm{a}}$ rodada quando a comparação foi entre o critério c1 (Tempo de efetivação da ação) e o critério c4 (Impacto da sensação de segurança). Quando indagado sobre esta indiferença, o decisor afirmou que para ele, neste caso a consequência de uma escolha ou outra não era tão significativa quando comparada com os outros julgamentos. Este é um aspecto interessante do procedimento, pois o decisor além de poder escolher entre as consequências hipotéticas dos critérios, também pode optar por não responder ou ser indiferente entre tais consequências. Assim, existe um 
ganho intrínseco de informações quando o decisor tem dificuldades de identificar sua preferência entre uma ação sobre outra, ou por não se sentir apto ou por simplesmente não desejar responder. A Tabela 3 apresenta a ordenação final das ações.

Tabela 3: Ranking das ações pelo FITradeoff.

\begin{tabular}{cc}
\hline Ranking & Ação \\
\hline 1 & a3 \\
2 & a7 \\
3 & a4 \\
4 & a8 \\
5 & a2 \\
6 & a5 \\
7 & a6 \\
8 & a1 \\
\hline
\end{tabular}

Fonte: Elaborada pelo autor.

O software do FITradeoff também forneceu o intervalo de valores para as constantes de escala, fornecendo os valores mínimo e máximo que cada constante pode assumir, conforme a Figura 2.

Figura 2: Intervalo de pesos dos critérios.

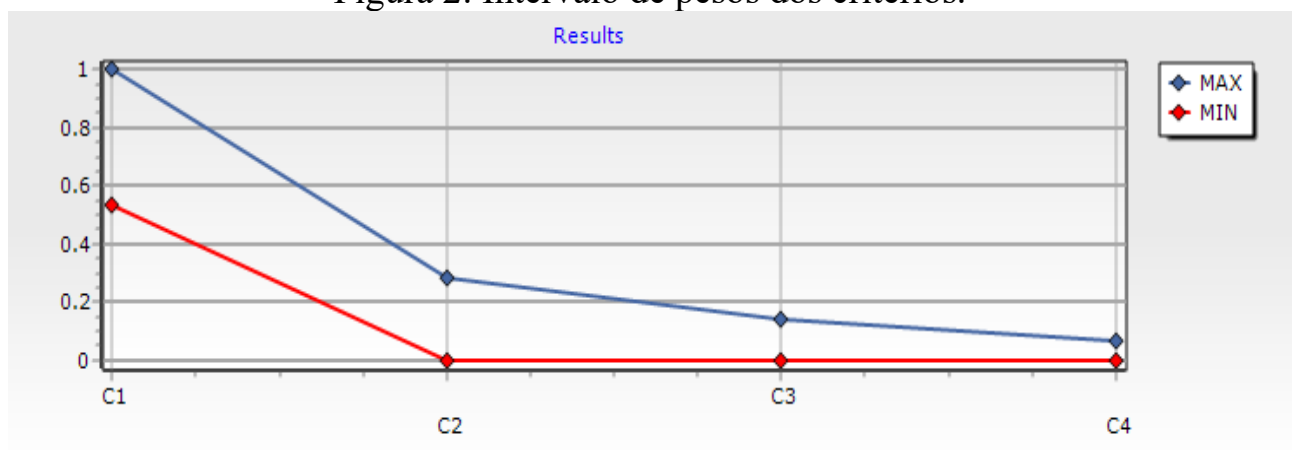

Fonte: Software do FITradeoff.

Dado que o procedimento trabalha com intervalos de pesos, qualquer valor de $k$, para seu respectivo critério, que esteja contido nos intervalos evidenciados pela Figura 2, a ordenação das ações será a mesma da ordenação apresentada na Tabela 3.

\subsection{Recomendações}

Portanto, ao fim da aplicação do método FITradeoff e de posse da ordenação final, é possível recomendar ao decisor as ações prioritárias. A ação a3 (Criar uma central especializada de apoio à violência contra a mulher) foi a ação mais bem colocada no ranking, sendo ela, portanto, que deve ser priorizada pelo decisor. Essa ação é seguida pela a7 (Fortalecer programas de reinserção de egressos do sistema prisional da cidade) e pela ação a4 (Aumentar o número de guardas municipais).

Nesse sentido, a gestão pública deve dedicar esforços e recursos para criar políticas públicas que viabilizem as ações supracitadas, de acordo com o ordenamento resultante da metodologia adotada.

\section{Discussões}

A adoção da ação prioritária a3 (Criar uma central especializada de apoio à violência contra a mulher) mostra-se coerente de acordo com a realidade vivenciada pelo Brasil. De 
acordo com o Anuário Brasileiro de Segurança Pública (2019) no ano de 2019 houve um crescimento de $11,30 \%$ em casos de feminicídio. Em $88,8 \%$ dos casos o autor do feminicídio foi o companheiro ou ex-companheiro da vítima. Das mulheres vítimas de violência, mais de $70 \%$ possuíam no máximo ensino fundamental.

No que diz respeito ao estado de Pernambuco, no ano de 2018, homicídios de vítimas do gênero feminino teve uma taxa de 4,6 por 100 mil mulheres, já o de feminicídio para o mesmo ano foi de 1,5 por 100 mil mulheres.

No contexto da pandemia causada pela COVID-19, de acordo com a Organização das Nações Unidas Women, além do perigo da doença causada pela COVID-19, existe uma preocupação latente no aspecto de violência contra a mulher. A pandemia ao impor o isolamento social, acarreta um maior contato das mulheres com seus parceiros violentos e ao mesmo tempo as privam do contato com outras pessoas e com os recursos que poderiam ajudálas a enfrentar este problema (ONU Women, 2020). Os estudos de Raj et al. (2020), Gulati e Kelly (2020), Moreira e Costa (2020) corroboram com a preocupação com o número de casos de violência doméstica em todo o mundo durante o período de pandemia pela COVID-19.

Esse fato pode ser confirmado de acordo com os dados divulgados pelo Anuário Brasileiro de Segurança Pública (2020a), em que destaca um crescimento de $22 \%$ no número de feminicídios no Brasil apenas nos meses de março e abril de 2020 em relação ao ano de 2019, período do início do confinamento pela pandemia. No estado de Pernambuco, no período de março até junho, período esse relativo a um isolamento social mais restrito, uma vez que ainda não tinham sido determinadas medidas de relaxamento no distanciamento social, o número de mulheres vítimas de violência doméstica chegou a marca de 11.417 vítimas, desse total, 6.058 correspondem a mulheres vítimas de violência residentes na região do interior do estado (SDSPE, 2020).

Portanto, a criação de uma central especializada de apoio à violência contra a mulher deve oferecer atendimentos e acolhimentos às mulheres vítimas de violência doméstica, bem como acolher seus filhos, observando com cautela a realidade do interior de Pernambuco no que diz respeito ao isolamento social imposto pela COVID-19. Essa central especializada deve concentrar todos os serviços públicos fundamentais para dar suporte às mulheres para que elas possam sair do ciclo de violência doméstica. Além disso, é importante oferecer a essas mulheres formas de subsistência, oferecendo, por exemplo, acesso a rede pública de saúde, assistência social à família e cursos profissionalizantes.

Portanto, outro ponto de preocupação deve ser o local mais indicado para a central especializada de apoio à violência contra a mulher, o município deve observar onde são as maiores recorrências destes tipos de violência e como mitigá-las de forma mais eficiente. Além disto, questionamentos sobre a causa deste tipo de violência devem ser investigados e tratados. Alguns dos possíveis motivos são o patriarcado estrutural, a segregação racial, de gênero e econômica, o que traz à tona as patologias sociais que devem ser erradicadas para que haja a minimização da violência doméstica.

No que diz respeito a ação a7 (Fortalecer programas de reinserção de egressos do sistema prisional da cidade) é importante criar condições para que ao término da pena, os egressos possam ter condições de retorno digno à sociedade, com uma nova perspectiva de vida e convívio social. Espera-se que com o fortalecimento de programas de reinserção seja possível como forma de reduzir a criminalidade, ou seja, que ao sair do sistema penitenciário o egresso não retorne a cometer crimes. De acordo com o Anuário Brasileiro de Segurança Pública (2019), no ano de 2017 havia um total de 706.619 presos no sistema penitenciário brasileiro (esse número não considera presos sob custódia das polícias em carceragens). Desse total, 31.001 correspondem a presos no sistema penitenciário do estado de Pernambuco.

Em períodos como o atual de pandemia da COVID-19, observa-se a importância deste tipo de política, visto que há uma superlotação carcerária na maioria das prisões brasileiras. Além disso, as instalações são precárias, em muitas unidades a assistência de saúde é mínima. Assim, é indispensável que haja ações para que os egressos não voltem ao sistema prisional, para que exista a possibilidade da redução nesse quantitativo de superlotação e minimizem as reincidências de crimes. 
Tal fato corrobora com o estudo de Tozzo et al. (2020) ao levantarem uma grande preocupação com o sistema prisional frente a pandemia causada pela COVID-19. Os autores destacam a importância de que durante a pena do indivíduo haja uma correção e a reabilitação do egresso do sistema prisional para que ele possa conviver na comunidade em uma vida legal.

O município, juntamente com outros órgãos públicos e privados devem preocupar-se com as formas adequadas para implementar maneiras objetivas e eficientes para que independente do período vivido, os cárceres tenham dignidade enquanto cumprem suas penas e tenham esperança de uma vida com possibilidades quando finalizarem suas sentenças, visto que observa-se a segregação, o preconceito e até a humilhação quando estes buscam oportunidades na sociedade, a qual em tese, deveria acolhê-los.

Já a ação a4 (Aumentar o número de guardas municipais) mostra-se como uma ação importante pois a aguarda municipal atua de maneira a inibir roubos, furtos, consumo de drogas, entre outros. Além disso, o aumento do efetivo de guardas aumenta a sensação de segurança de população e inibi possíveis ações criminosas contra a sociedade.

No que diz respeito a metodologia adotada, o uso do método FITradeoff para ordenação mostrou-se satisfatório ao contexto abordado nesse estudo. Devido à falta de informações e hesitações por parte do decisor na tomada de decisão, além do tempo reduzido, o método FITradeoff apresentou vantagens quanto ao seu uso: por ser um processo de elicitação flexível, o FITradeoff requer menos esforço do decisor; utiliza informações parciais e não completas, minimizando também o esforço cognitivo do decisor; as perguntas respondidas pelo decisor carregam menos incertezas ou hesitações; possibilidade do decisor interromper o processo antes do final da elicitação; o uso do software do FITradeoff fornece informações que podem ser visualizadas por gráficos, facilitando assim a compreensão do decisor (de Almeida et al., 2016; Gusmão e Medeiros, 2016; de Almeida-Filho et al., 2017; Carrillo et al., 2018; Roselli et al., 2019; Frej et al., 2019).

Como visto anteriormente, o uso do FITradeoff reduz as inconsistências e o esforço cognitivo necessário do decisor durante todo o processo. Ao invés de uma avaliação de aproximadamente $3(\mathrm{n}-1)$ relações de indiferença para o estabelecimento das constantes de escala, conforme o procedimento tradicional de tradeoff (de Almeida et al., 2016), o que nesse estudo corresponderia a 9 ciclos, por meio do FITradeoff foram realizados apenas 4 ciclos, reduzindo o tempo, o esforço cognitivo do decisor e a quantidade de perguntas necessárias para obter o ordenamento das ações.

\section{Considerações Finais.}

Esse estudo teve como objetivo definir e priorizar ações estratégicas de segurança pública a fim de reduzir a criminalidade durante o período da pandemia causada pela COVID-19 em um município do interior do estado de Pernambuco. Por meio das metodologias VFT e FITradeoff de ordenação foi possível recomendar ao decisor do problema ações estratégicas que devem ser encaradas como prioridades para garantia da segurança pública do município.

Do ponto de vista teórico esse estudo contribui para a literatura sobre o tema da COVID19, evidenciando a viabilidade e importância da aplicabilidade de metodologias de suporte à decisão no contexto da pandemia. Portanto, pesquisadores não apenas da área de segurança pública, como de outras áreas e municípios, podem replicar a metodologia trazida nesse estudo com as devidas adaptações ao contexto observado. Já do ponto de vista prático, esse estudo contribui, de maneira estruturada, a auxiliar os gestores públicos no estabelecimento de políticas públicas de segurança durante o período de crise causado pela pandemia da COVID-19, principalmente durante o isolamento social. É importante que os gestores públicos, além de medidas de saúde pública, busquem minimizar a criminalidade, principalmente no ambiente doméstico durante o isolamento social.

Uma das principais limitações do trabalho é o fato do tema da COVID-19 ser recente, fato esse que traz para a literatura uma carência de estudos que abordem a questão de segurança pública no contexto da pandemia. Apesar da literatura sobre a COVID-19 ter ganhado força, a grande maioria dos seus estudos abordam aspectos de saúde e/ou comportamentais. Ainda há 
uma carência de estudos que tratem questões como segurança pública e mobilidade, por exemplo, nesse contexto da pandemia. Os autores acreditam que esse trabalho possa servir de base, como um movimento inicial para estudos futuros para que haja uma melhor compreensão do tema de segurança pública frente a pandemia da COVID-19 para outros pesquisadores da área.

Por fim, como a maioria das decisões dentro da esfera pública devem refletir as preferências de diversos grupos e a necessidade de justificar a decisão, sugere-se para trabalhos futuros o uso da metodologia no contexto de decisão em grupo para priorização de estratégias de segurança pública. Além disso, realizar um estudo de viabilidade da adoção das ações consideradas prioritárias frente a um cenário caracterizado por restrições de recursos, como as financeiras.

\section{Referências}

Alencar, M. H., Priori Jr., L. e Alencar, L. H. Structuring objectives based on value-focused thinking methodology: Creating alternatives for sustainability in the built environment. Journal of Cleaner Production, v. 156, p. 62-73, 2017.

AlMaian, R. Y., Needy, K. L., Alves, T. C. L. e Walsh, K. D. Analyzing effective supplierquality-management practices using simple multiattribute rating technique and value-focused thinking. Journal of Management in Engineering, v. 32, n. 1, p. 04015035, 2016.

Anuário Brasileiro de Segurança Pública 2019. 2019. Disponível em: http://www.forumseguranca.org.br/wp-content/uploads/2019/10/Anuario-2019-

FINAL_21.10.19.pdf. Acesso em: 14/05/2020.

Anuário Brasileiro de Segurança Pública 2020. 2020a. Disponível em: https://forumseguranca.org.br/publicacoes_posts/violencia-domestica-durante-pandemia-decovid-19-edicao-02. Acesso em: 27/07/2020.

Anuário Brasileiro de Segurança Pública 2020. 2020b. Disponível em: http://dados.mj.gov.br/dataset/210b9ae2-21fc-4986-89c62006eb4db247/resource/feeae05e-faba406c-8a4a-512aec91a9d1/download/indicadoressegurancapublicaufmar20.xlsx. Acesso em: $20 / 08 / 2020$.

Belton, V. e Stewart, T. J. Multicriteria Decision Analysis: An Integrated Approach. Kluwer Academic Publishers, 2002.

Brasil. Constituição da República Federativa do Brasil de 1988. Disponível em: $<$ https://www.senado.leg.br/atividade/const/con1988/con1988_26.06.2019/art_144_.asp>.

Acesso em: 14/05/2020.

Carrillo, P.A.A., Roselli, L.R.P., Frej, E.A. e de Almeida, A.T. Selecting an agricultural technology package based on the flexible and interactive tradeoff method. Annals of Operations Research, 2018. https://doi.org/10.1007/s10479-018-3020-y

de Almeida, A. T., de Almeida, J. A., Costa, A. P. C. S. e de Almeida-Filho, A. T. A new method for elicitation of criteria weights in additive models: Flexible and interactive tradeoff. European Journal of Operational Research, v. 250, n. 1, p. 179-191, 2016.

de Almeida-Filho, A. T., de Almeida, A. T. e Costa, A. P. C. S. A flexible elicitation procedure for additive model scale constants. Annals of Operations Research, v. 259, n. 1, p. 65-83, 2017. 
Fossile, D. K., Frej, E. A., da Costa, S. E. G., de Lima, E. P. e de Almeida, A. T. Selecting the most viable renewable energy source for Brazilian ports using the FITradeoff method. Journal of Cleaner Production, v. 260, 2020. https://doi.org/10.1016/j.jclepro.2020.121107

Frej, E. A., Roselli, L. R. P., de Almeida, J. A. e de Almeida, A. T. A multicriteria decision model for supplier selection in a food industry based on FITradeoff method. Mathematical Problems in Engineering, v. 2017, 2017. https://doi.org/10.1155/2017/4541914

Frej, E. A., de Almeida, A. T. e Costa, A. P. C. S. Using data visualization for ranking alternatives with partial information and interactive tradeoff elicitation. Operational Research, v. 19, p. 909-931, 2019.

Gulati, G. e Kelly, B. D. Domestic violence against women and the COVID-19 pandemic: What is the role of psychiatry? International Journal of Law and Psychiatry, v. 71, 2020. https://doi.org/10.1016/j.ijlp.2020.101594

Gusmão, A. P. H. e Medeiros, C. P. A model for selecting a strategic information system using the FITradeoff. Mathematical Problems in Engineering, v. 2016, 2016.

https://doi.org/10.1155/2016/7850960

Höfer, T. e Madlener, R. A participatory stakeholder process for evaluating sustainable energy transition scenarios. Energy Policy, v. 139, 2020. https://doi.org/10.1016/j.enpol.2020.111277

Itiki, R. e Chowdhury, P. R. Fast deployment of COVID-19 disinfectant from common ethanol of gas stations in Brazil. Health Policy and Technology, v. 9, n. 3, p. 384-390, 2020.

Keeney, R. L. Value Focused Thinking: A Path to Creative Decision Making. Cambridge MA: Harvard University Press, 1992.

Keeney, R. L. Creativity in decision making with value focused thinking. Sloan Management Review, v. 35, p. 33-41, 1994.

Keeney, R. L. Value-focused thinking: Identifying decision opportunities and creating alternatives. European Journal of Operational Research, v. 92, n. 3, p. 537-549, 1996.

Keeney, R.L. e Raiffa, H. Decisions with Multiple Objectives: Preferences and Value Tradeoffs. New York, 1976.

Lesinski, G. Application of value focused thinking and fuzzy systems to assess system architecture. Procedia Computer Science, v. 61, p. 168-175, 2015.

Merrick, J. R. W. e Grabowski, M. Decision performance and safety performance: A valuefocused thinking study in the oil industry. Decision Analysis, v. 11, n. 2, p. 105-116, 2014.

Morais, D. C., Alencar, L. H., Costa, A. P. C. S. e Keeney, R. L. Using value-focused thinking in Brazil. Pesquisa Operacional, v. 33, n. 1, p. 73-88, 2013.

Moreira, D. N. e Costa, M. P. The impact of the Covid-19 pandemic in the precipitation of intimate partner violence. International Journal of Law and Psychiatry, v. 71, 2020. https://doi.org/10.1016/j.ijlp.2020.101606

ONU Women - Organização das Nações Unidas Women. 2020. Violence against women and girls: the shadow pandemic. Disponível em: 
https://www.unwomen.org/en/news/stories/2020/4/statement-ed-phumzile-violence-againstwomen-during-pandemic $>$. Acesso em: 30/07/2020.

OMS - Organização Mundial da Saúde. 2020. Disponível em: https://www.who.int/dg/speeches/detail/who-director-general-s-opening-remarks-at-the-mediabriefing-on-covid-19---11-march-2020. Acesso em: 31/07/2020.

Pergher, I., Frej, E. A., Roselli, L. R. P. e de Almeida, A. T. Integrating simulation and FITradeoff method for scheduling rules selection in job-shop production systems. International Journal of Production Economics, v. 227, 2020. https://doi.org/10.1016/j.ijpe.2020.107669

Poleto, T., Clemente, T. R. N., Gusmão, A. P. H., Silva, M. M. e Costa, A.P.C.S. Integrating value-focused thinking and FITradeoff to support information technology outsourcing decisions. Management Decision, 2020. https://doi.org/10.1108/MD-09-2019-1293

Raj, A., Johns, N. E., Barker, K. M. e Silverman, J. G. Time from COVID-19 shutdown, gender-based violence exposure, and mental health outcomes among a state representative sample of California residentes. EClinicalMedicine, v. 26, 2020. https://doi.org/10.1016/j.eclinm.2020.100520

Rodriguez-Morales, A. J., Gallego, V., Escalera-Antezana, J. P., Méndez, C. A., Zambrano, L. I., Franco-Paredes, C., et al. COVID-19 in Latin America: The implications of the first confirmed case in Brazil. Travel Medicine and Infectious Disease, v. 35, 2020. https://doi.org/10.1016/j.tmaid.2020.101613

Roselli, L. R. P., de Almeida, A. T. e Frej, E. A. Decision neuroscience for improving data visualization of decision support in the FITradeoff method. Operational Research, v. 19, p. 933-953, 2019.

SDS-PE - Secretaria de Defesa Social do estado de Pernambuco. 2020. Estatísticas. Disponível em: http://www.sds.pe.gov.br/estatisticas. Acesso em: 31/07/2020.

Sinesp - Sistema Nacional de Informações de Segurança Pública. 2020. Disponível em: http://dados.mj.gov.br/dataset/sistema-nacional-de-estatisticas-de-seguranca-publica. Acesso em: $14 / 05 / 2020$.

Smith, C. M. e Shaw, D. The characteristics of problem structuring methods: A literature review. European Journal of Operational Research, v. 274, n. 2, p. 403-416, 2019.

Tozzo, P., D'Angiolella, G. e Caenazzo, L. Prisoners in a pandemic: We should think about detainees during Covid-19 outbreak. Forensic Science International: Synergy, v. 2, p. 162-163, 2020. 